\title{
Gerador de Plasma frio de baixo custo
}

\author{
Alves, D. P. ${ }^{1 *}$; Pinheiro, C. J. G. ${ }^{2}$; Moises C.R ${ }^{3}$; Passamai J. L. ${ }^{4}$; Melo, D. C. A. ${ }^{3}$ \\ 1 Programa de Pós-Graduação em Engenharia Química, Universidade Federal do Espírito Santo, Alegre, ES, Brasil. \\ 2 Departamento de Engenharia Rural, Universidade Federal do Espírito Santo, Alegre, ES, Brasil. \\ 3 Departamento de Química e Física, Universidade Federal do Espírito Santo, Alegre, ES, Brasil. \\ 4 Departamento de Física, Universidade Federal do Espírito Santo, Vitória, ES, Brasil. \\ *e-mail: douglas.palves15@gmail.com
}

\begin{abstract}
Resumo
Este trabalho visa a construção de um dispositivo gerador de plasma frio à pressão atmosférica. Este dispositivo além de compacto, com facilidade de transporte, possui uma boa relação custo benefício devido ao uso de materiais de baixo custo. $O$ objetivo deste projeto foi construir um gerador de plasma utilizando materiais de baixo custo. Constituído basicamente por uma fonte de alta tensão com frequência e tensão ajustáveis para a ionização do gás utilizado, um braço suporte que pode ser ajustado à diferentes alturas e um dispositivo para a geração do plasma frio à pressão atmosférica. Utilizando gás argônio foi possível gerar plasma frio com um fluxo intenso e direcionável.
\end{abstract}

Palavras chaves: Gerador de plasma, Plasma frio, Baixo custo.

\begin{abstract}
This work aims at the construction of a cold plasma generator at atmospheric pressure. This device, besides being compact and easy to transport, is cost effective due to the use of low cost materials. The goal of this project was to build a plasma generator using low cost materials. Basically constituted by a high voltage source with adjustable frequency and voltage for the ionization of the gas used, a support arm that can be adjusted to different heights and a device for the generation of cold plasma at atmospheric pressure. Using argon gas, it was possible to generate cold plasma with an intense and directional flow.
\end{abstract}

Keywords; Plasma reactor, Cold plasma, Low, cost.

\section{Introdução}

O termo "plasma" é usado em Física para descrever um gás eletricamente condutor, este gás é constituído por partículas livres carregadas e não carregadas, íons, elétrons, nêutrons, átomos e moléculas que exibem um comportamento coletivo graças às forças coulombianas $[1,2]$. O plasma foi identificado pela primeira vez em 1879 pelo físico inglês Wiliam Crookes como um novo estado da matéria, porém, foi somente em 1929 que o cientista americano Irving Langmuir utilizou o termo "plasma" ao tratar de um gás ionizado [2].

Devido às suas características, o plasma é considerado "O quarto estado da matéria" [1-3]. Apesar de ser o menos conhecido dos estados da matéria, o plasma é o estado mais abundante do universo sendo encontrado em uma ampla variedade de pressão e temperatura. Cerca de $99 \%$ do universo conhecido é constituído de plasma [2,3].

Devido a sua maleabilidade os plasmas são empregados desde reatores de fusão nuclear [3] a aplicações biomédicas [4].

Em um ponto de vista mais amplo, podemos categorizar o plasma em dois tipos principais, plasma natural e plasma de laboratório [3]. O plasma natural é o tipo mais presente em todo o universo, podemos citar como exemplo a corona solar, a aurora boreal, os ventos solares, as nebulosas e a maioria dos objetos astronômicos [2,3]. Os plasmas de laboratório ou plasmas artificiais são plasmas produzidos por processos não naturais e podem ser classificados como plasmas físicos (relacionados à fusão termonuclear), plasmas térmicos (plasmas de altas temperaturas usados principalmente para cortes e soldagem de metais) e plasmas frios (plasmas à temperatura ambiente) $[3,5,6]$.

De uma maneira geral, o plasma pode ser produzido adicionando-se energia térmica ou elétrica às partículas constituintes do gás, o objetivo é proporcionar a dissociação de elétrons dos átomos ou moléculas [5]. 0 método de produção do plasma varia de acordo como tipo de plasma que se deseja produzir. Nos plasmas térmicos e frios é geralmente utilizado alta tensão de maneira pulsada $[3,6]$.

Ao se aplicar alta tensão de maneira pulsada entre dois eletrodos metálicos, em especial se inserido parcialmente um dielétrico entre eles, é produzido 
plasma por um processo chamado de descarga em barreira dielétrica (DBD) [6,7]. Deste modo são geradas microdescargas por cargas elétricas que se acumulam na superfície do dielétrico, rompendo a rigidez dielétrica do gás e do dielétrico [6,7]. A ruptura da rigidez dielétrica do gás que se encontra entre os eletrodos produz várias microdescargas que surgem em pontos aleatórios na região entre os eletrodos ionizando o gás e gerando o plasma DBD $[1,6,7]$.

O plasma frio atmosférico possui diversas aplicações como o esterilização de superfícies e materiais (em especial materiais termosenssíveis), controle de poluentes do ar, deposição de filmes finos, modificação de superfícies, e mais recentemente tem-se dado bastante atenção as suas aplicações biomédicas que incluem inativação microbiana, coagulação do sangue, descontaminação de equipamentos médicos, cicatrização de feridas e aplicações odontológicas [7,8]. Neste projeto foram construídos uma fonte de alta voltagem (HV) com controle de tensão e frequência, braço de suporte ajustável e o dispositivo gerador de jato de plasma, com o objetivo de se produzir plasma frio à pressão atmosférica para aplicações biomédicas.

\section{Materiais e Métodos}

\subsection{Fonte de alta tesão (HV)}

A fonte de alta tensão pulsada teve como referência o trabalho de $[6,9]$. Foram realizadas alterações na fonte como a substituição do transformador original e o regulador para os cooler por uma fonte (12V) de computador usada, com a substituição dessas peças e a compra dos demais componentes que são de fácil montagem, o valor da fonte ficou estimado em 300 reais. Foram encontrados modelos comerciais com as mesmas características em valores que variam entre 10 mil a 20 mil reais. A carcaça da fonte foi confeccionada em madeira e possui as dimensões 30 $\mathrm{cm} \times 30 \mathrm{~cm} \times 15 \mathrm{~cm}$ [10].

\subsection{Braço Suporte}

Para o braço e suporte foi utilizada uma base de luminária (foi usada uma luminária da marca LLUM ${ }^{\circledR}$ ), Foi escolhido este objeto por seu baixo custo e por ser ajustável à diferentes configurações podendo então direcionar o jato de plasma, sua base foi facilmente fixada sobre a parte superior da fonte HV, e sua extremidade superior depois de removido o bocal de fixação da lâmpada o dispositivo gerador de plasmas foi facilmente fixado. $O$ cabo de alta tensão passa pelo interior do corpo da base da luminária [10].

\subsection{Dispositivo gerador de plasma frio}

O dispositivo gerador de plasma frio foi confeccionado em TEFLON, este material é de baixo custo e bem maleável. Para a construção do dispositivo foi usado como referência o trabalho de [6], realizando algumas modificações como a substituição do eletrodo central por um tubo de cobre [10].

\section{Resultados e Discussões}

Controlando os parâmetros elétricos (tensão e frequência aplicada na faixa de $0 \mathrm{KV}$ a $20 \mathrm{KV}$ e de 500 $\mathrm{Hz}$ a 1,29 KHz respectivamente), o fluxo de gás (vazão controlada por um manômetro na saída do cilindro de argônio, de $0 \mathrm{~L} / \mathrm{min}$ a $25 \mathrm{~L} / \mathrm{min}$ ) e a distância de tratamento da amostra $(0 \mathrm{~cm}$ a $5 \mathrm{~cm})$, foi possível variar a intensidade do plasma gerado. O controle da intensidade pode aumentar ou diminuir a produção de radicais livres no plasma. Um fato importante a ser ressaltado é que o gerador de plasma não apresentou fugas de corrente. Ao se trabalhar com a alta tensão muitas das vezes ocorre a presença e formação de fugas elétrica (arcos) em lugares indesejados. Isto ocasiona perdas elétricas e a possibilidade de choque elétrico ao usuário, fato não observado neste projeto. $\mathrm{O}$ gerador é mostrado na figura 1.

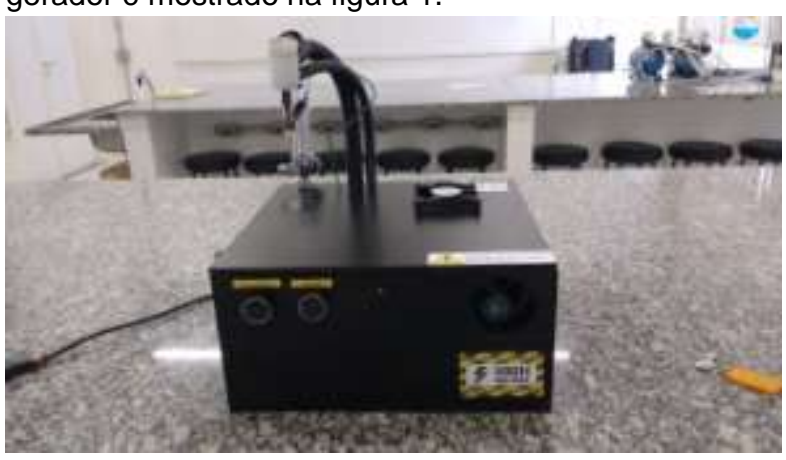

Figura 1: Gerador de plasma frio à pressão atmosférica

Sob certas condições, as descargas produzidas pela técnica DBD podem operar em regime filamentar e/ou difuso. O regime filamentar e caracterizado pela formação de pequenos arcos elétricos no plasma, enquanto no regime difuso o plasma é mais uniforme. Graças à sua uniformidade este plasma é o mais vantajoso para tratamento e esterilização de materiais e é produzido mais facilmente através do ajuste de frequências mais altas (dezenas de $\mathrm{kHz}$ ) e menor pressão atmosférica [6,7]. Os parâmetros, tensão aplicada entre os eletrodos da ordem de $\sim 18 \mathrm{KV}$, frequência de $1,29 \mathrm{KHz}$ e vazão do gás argônio 4 L/min, foram capazes de produzir plasma frio a pressão atmosférica no regime difuso como observado na figura 2. Este regime difuso pode ser classificado através de análises visuais, estes parâmetros geraram um jato de plasma de maior comprimento, de $\sim 2 \mathrm{~cm}$.

\section{Conclusão}

Grande atenção tem-se dado ao plasma frio atmosférico nos últimos anos, principalmente na área de biomedicina como agente esterilizante. Neste trabalho construímos um gerador de plasma frio à pressão à atmosférica, seguro por não apresentar fugas elétricas, utilizando materiais de baixo custo. As 
configurações, tensão aplicada entre os eletrodos da ordem de $\sim 18 \mathrm{KV}$, frequência de $1,29 \mathrm{KHz}$ e vazão do gás argônio $4 \mathrm{~L} / \mathrm{min}$, foram capazes de produzir jatos de plasma da ordem de $2 \mathrm{~cm}$, valor segundo a literatura [4] aplicável em biomedicina na inativação de baterias fungos dentre outros microrganismos.

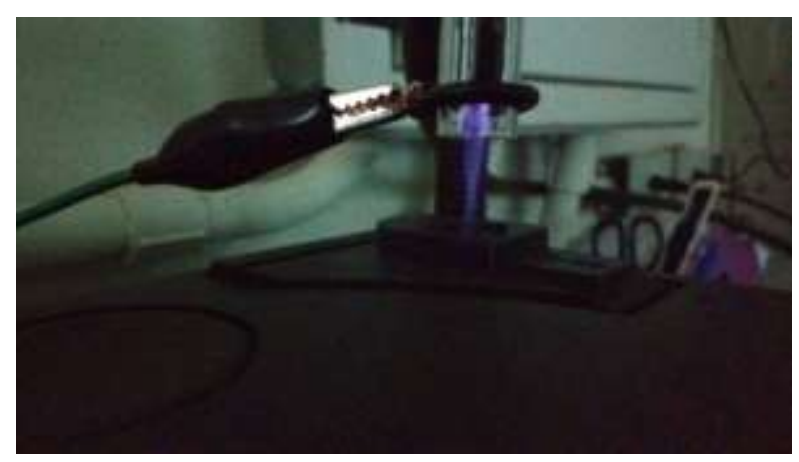

Figura 1: Jato de plasma frio a pressão atmosférica

\section{Agradecimentos}

Os autores agradecem a CAPES pelo apoio financeiro.

\section{Referências}

[1] SANTOS, A. C. P. Redução aluminotérmica do óxido de tântalo usando uma tocha de plasma como ignitor. Tese (Doutorado em Engenharia de materiais), Programa de Pós-Graduação em Ciências e Engenharia de Materiais, Centro de Ciências Exatas e da Terra, Universidade Federal do Rio Grande do Norte, Natal, Rio grande do Norte, 2007.

[2] FARIAS, E. E. Estudo do Plasma produzido por descarga elétrica no limiar da transição Townsend "glow". Dissertação (Mestrado em Plasmas em Ciências Físicas), Programa Pós-Graduação em Física, Universidade Federal Fluminense, Niterói, Rio de Janeiro, 2006.

[3] FELIPINI, C. L. Noções sobre plasma térmico e suas principais aplicações. Usjt integração. № 41. p. 147-151, 2005.
[4] KOLB, J. F.; et al. Cold atmospheric pressure air plasma jet for medical applications. Journal Applied Physics Letters. applied physics letters 92, 241501, 2008

[5] GASPERI, J. I. Aplicações do plasma térmico e descarga corona para eliminação de resíduos ambientalmente perigosos. (Trabalho de Conclusão de Curso de Química), Departamento de Química, Universidade Federal de Santa Catarina, Florianópolis, Santa Catarina, 2008.

[6] NASCIMENTO NETO, A. B. Desenho e Construção de um Protótipo Gerador de Jato de Plasma Frio à Pressão Atmosférica para Aplicações Biomédicas. Dissertação (Mestrado em Engenharia Mecânica), Programa de Pós-Graduação em Engenharia Mecânica, Universidade Federal do Rio Grande do Norte, Natal, Rio grande do Norte, 2013.

[7] ROCHA, V. Esterilização de materiais termossensíveis através de aplicação de plasma gerado por descarga com barreira dielétrica (DBD). Dissertação (Mestrado em Física Aplicada a Tecnológica de Plasma), Faculdade de Engenharia do Campus de Guaratinguetá, Universidade Estadual Paulista, Guaratinguetá, São Paulo, 2009.

[8] NISHIME, T. M. C.; et al. Non-thermal atmospheric pressure plasma jet applied to inactivation of different microorganisms. Surface \& Coatings Technology 312 (2017) 19-24, 2016

[9] BRAGA, N. C; Motor iônico, saber eletrônica, 1986.

[10] ALVES, D. P. Construção de um reator de plasma frio à pressão atmosférica. Trabalho de Conclusão de curso (Licenciatura em Física), Centro de Ciências Exatas, Naturais e da Saúde, Universidade Federal do Espírito Santo, Alegre, Espírito Santo, 2016. 